\title{
Nachhaltige Regionalentwicklung in Naturparken - 10-Jahres-Rückblick und Zukunftsperspektiven
}

\author{
Florian Weber, Jörg Liesen, Friedericke Weber, Nora Crossey \\ Eingegangen: 9. Februar 2021 - Angenommen: 31. Mai 2021 - Online veröffentlicht: 10. Juni 2021
}

\begin{abstract}
Zusammenfassung
Die Verankerung der nachhaltigen Regionalentwicklung als gesetzliche Aufgabe für die über 100 Naturparke in Deutschland im Bundesnaturschutzgesetz im Jahr 2002 führte zu neuen Impulsen in der Naturparkarbeit, wie quantitative und qualitative Erhebungen für die Jahre 2010 und 2011 ergaben. Neben Chancen zur Profilbildung wiesen die Analysen gleichzeitig auf Herausforderungen hin, die einer fundierten perspektivischen Aufgabenerfüllung hinderlich gegenüberstehen könnten. Forschungs- und gleichzeitig praxisorientiert wird auf der Grundlage einer erneuten standardisierten Umfrage auf gleicher Befragungsbasis wie 2011 bei den Naturparken der Frage nachgegangen, wie im Jahr 2020 deren Geschäftsführungen bzw. Verwaltungen die Potenziale und Restriktionen der Aufgabe der nachhaltigen Regionalentwicklung im Portfolio ihrer Gesamtzielsetzung und ihrer Ausrichtungen bewerten. Aus
\end{abstract}

\footnotetext{
$\square$ Jun.-Prof. Dr. Florian Weber, Fachrichtung Gesellschaftswissenschaftliche Europaforschung, Campus, Universität des Saarlandes, 66123 Saarbrücken, Deutschland

florian.weber@uni-saarland.de
}

Jörg Liesen, Verband Deutscher Naturparke e.V., HolbeinstraBe 12, 53175 Bonn, Deutschland liesen@naturparke.de

Dr. Friedericke Weber, Biosphärenreservat Pfälzerwald-Nordvogesen, Geschäftsstelle Pfälzerwald, Franz-Hartmann-Straße 9, 67466 Lambrecht (Pfalz), Deutschland f.weber@pfaelzerwald.bv-pfalz.de

Nora Crossey, Fachrichtung Gesellschaftswissenschaftliche Europaforschung, Campus, Universität des Saarlandes, 66123 Saarbrücken, Deutschland

nora.crossey@uni-saarland.de

(c) (1) () 2021 Weber; licensee oekom verlag. This Open Access article is published under a Creative Commons Attribution 4.0 International License. einer konzeptionellen Perspektive heraus zeigt sich, dass die Naturparke mit ihrem vernetzenden Handeln zentralen Kriterien der Regional-governance-Debatte entsprechen. Konzeptionell und praktisch eingeordnet, verbleiben Unschärfen, was unter ,nachhaltige Regionalentwicklung zu subsumieren ist, eng gekoppelt an die Begriffsweite von ,Nachhaltigkeit‘. Anwendungsorientiert unterstreichen die Befragungsergebnisse im Zeitvergleich gleichzeitig, dass in den NaturparkGeschäftsführungen/-verwaltungen in vielen Bereichen positive Entwicklungsperspektiven konstatiert werden. Der Prozess der Naturparke in Deutschland Richtung Regionalmanager in ,Modellregionen nachhaltiger Entwicklung' schreitet damit weiter voran.

Schlüsselwörter: Naturparke - nachhaltige Regionalentwicklung - Aufgabenportfolio Herausforderungen - Chancen

\section{Sustainable regional development in nature parks - 10-year retrospection and future prospects}

\begin{abstract}
In 2002, sustainable regional development was introduced as a legal mandate in the Federal Nature Conservation Act (Bundesnaturschutzgesetz) for the more than 100 nature parks in Germany, subsequently expanding the scope of their activities and introducing new incentives. Quantitative and qualitative surveys for 2010 and 2011 indicated new opportunities for nature parks to develop distinct profiles, as well as challenges regarding future task fulfilment. In a pursuit of both research- and practice-oriented questions and based on an adapted replication of these surveys, we ask how the management evaluate potentials and restrictions related to the task of sustainable regional development in the context of their
\end{abstract}


overall portfolio of tasks and objectives. From a conceptual perspective, it becomes evident that due to their networking activities, nature parks correspond to central criteria of the regional governance debate. Nonetheless, a certain unclarity remains with regard to the notion of 'sustainable regional development, which is closely linked to the similarly broad concept of 'sustainability'. From an application-oriented perspective, the survey results show that nature park managers perceive positive development prospects in many areas. Nature parks in Germany have thus proceeded further on the path towards a role as regional managers in 'model regions of sustainable development'.

Keywords: Nature parks = sustainable regional development - task portfolio - threats - opportunities

\section{Einleitung: Naturparke und die Aufgabe der nachhaltigen Regionalentwicklung}

Naturparke sind die ältesten Großschutzgebiete in Deutschland. Zusammen mit Nationalparken und Biosphärenreservaten zielen sie auf einen großflächigen Gebietsschutz ab. Bereits 1906 fanden sich in der Lüneburger Heide private Initiativen, die 1909 in den ,Verein Naturschutzpark' mündeten, um die dortige, als schützenswert angesehene Landschaft zu bewahren (Lommel 1974: 96f.). Eine größere Dynamik zur Gründung von Naturparken fällt allerdings erst in die Nachkriegszeit: 1956 wurde durch den Hamburger Großkaufmann Alfred Toepfer das ,Naturparkprogramm“ vorgestellt, mit dem die Einrichtung von mindestens 25 Parken für Naturschutz und Erholung eingefordert wurde (vgl. Forst 2019: 374). Zum entscheidenden Zugang wurde dabei die proklamierte Gleichwertigkeit von Natur- und Landschaftsschutz auf der einen und der gesellschaftlichen Erholungsfunktion auf der anderen Seite (Liesen/Köster/Porzelt 2008: 26), worüber sich auch die große Zustimmung für dessen Idee erklären lässt. Die Einrichtung der Parke erfolgte ab 1957 vorrangig zwischen den großen Siedlungs- und Industrieregionen, sodass statt eines ,einseitigen Bewahrungsgedankens ' auch die aktive Erholungsnutzung für die urbane Bevölkerung Berücksichtigung fand (Job/Metzler 2006: 355; Schöbel 2018: 12) - also als ein Schwerpunkt zugunsten der Erholungsvorsorge (Scherfose 2019: 429). Orientierungsgebend wurde dabei auch ein Gutachten des Instituts für Raumforschung, in dem Gerhard Isbary (1959) zentrale Leitlinien darlegte, die im Hinblick auf die Auswahl und Neugründung von Naturparken Beachtung finden sollten (vgl. Job 1991: 70 ff.). Im Zuge der Etablierung der ersten Nationalparke in Deutschland (1970: Bayerischer Wald, 1978: Berchtesgaden) - inspiriert von US-amerikanischen
Entwicklungen -, die durch die Einbettung in den internationalen Kontext exklusiver erschienen und forciert durch den Wegfall von Bundeszuschüssen für Naturparke im Zuge der Verabschiedung des ersten nunmehr rahmengesetzgebenden Bundesnaturschutzgesetzes 1976, zeigte sich eine sinkende Wertschätzung der Naturpark-Grundidee (Weber 2013: 46f.; Majewski/Engelbauer/Job 2019: 422). Doch nach der Wiedervereinigung und Novellierungen des Bundesnaturschutzgesetzes 2002 und 2017 ergaben sich neue Perspektiven und ein Anwachsen auf 103 Naturparke (Stand 2021), die inzwischen über 28 Prozent der Landesfläche der Bundesrepublik Deutschland umfassen (vgl. Abbildung 1) und so eine hohe Reichweite entfalten (Forst 2019; Weber/ Weber 2019; Mayer/Stoll-Kleemann 2020). ${ }^{1}$

Seit 2002 ist mit $\S 27$ Abs. 1 Nr. 6 BNatSchG ${ }^{2}$ die nachhaltige Regionalentwicklung als gesetzliche Aufgabe für Naturparke verankert, die die ,klassischen “ Aufgabengebiete Naturschutz, Landschaftspflege, Erholung und Tourismus ergänzt. Hierüber zeichneten sich neue Impulse für die Parke ab, wie Weber (2013) ausführlich im Rahmen ihrer Promotion mit einem quantitativen und ergänzend qualitativen Ansatz für die Jahre 2010 und 2011 herausgearbeitet hat. Neben Chancen zur Profilbildung wiesen die Erhebungen zudem auf (fortbestehende) Herausforderungen hin, die einer fundierten perspektivischen Aufgabenerfüllung hinderlich gegenüberstehen könnten. Forschungs- und gleichzeitig praxisorientiert stellt sich vor diesem Hintergrund die Frage, wie zehn Jahre später die Geschäftsführungen bzw. Verwaltungen der deutschen Naturparke die Potenziale und Restriktionen der Aufgabe der nachhaltigen Regionalentwicklung im Portfolio ihrer Gesamtzielsetzung und ihrer Ausrichtungen bewerten. Zur Einordnung wurde im Jahr 2020 in einer Kooperation aus der Arbeitsgruppe Europastudien an der Universität des Saarlandes, dem Verband Deutscher Naturparke e.V. (VDN) und Friedericke Weber die standardisierte Befragung aller NaturparkGeschäftsführungen wiederholt.

Im Folgenden skizzieren wir zentrale Ergebnisse dieser neuerlichen Erhebung im Vergleich zu der aus 2011, basierend auf einer grundlegenden Einordnung und Rahmung über die Aufgabensetzung, nachhaltiger Regionalentwicklung' im Großschutzgebietskontext (Kapitel 2). Auf diese Weise kann zum einen eine ,Positionsbestimmung' sowie ein ,Positionsabgleich“ der komplexen Aufgabe erfolgen und zum anderen in anwendungsbezogener Ausrichtung den Naturpark-Akteuren dargelegt werden, wo weitere Hand-

\footnotetext{
1 Vgl. https://www.bfn.de/themen/gebietsschutzgrossschutzgebiete/ naturparke.html (25.05.2021).

2 Bundesnaturschutzgesetz vom 29. Juli 2009, das zuletzt durch Artikel 5 des Gesetzes vom 25. Februar 2021 geändert worden ist.
} 


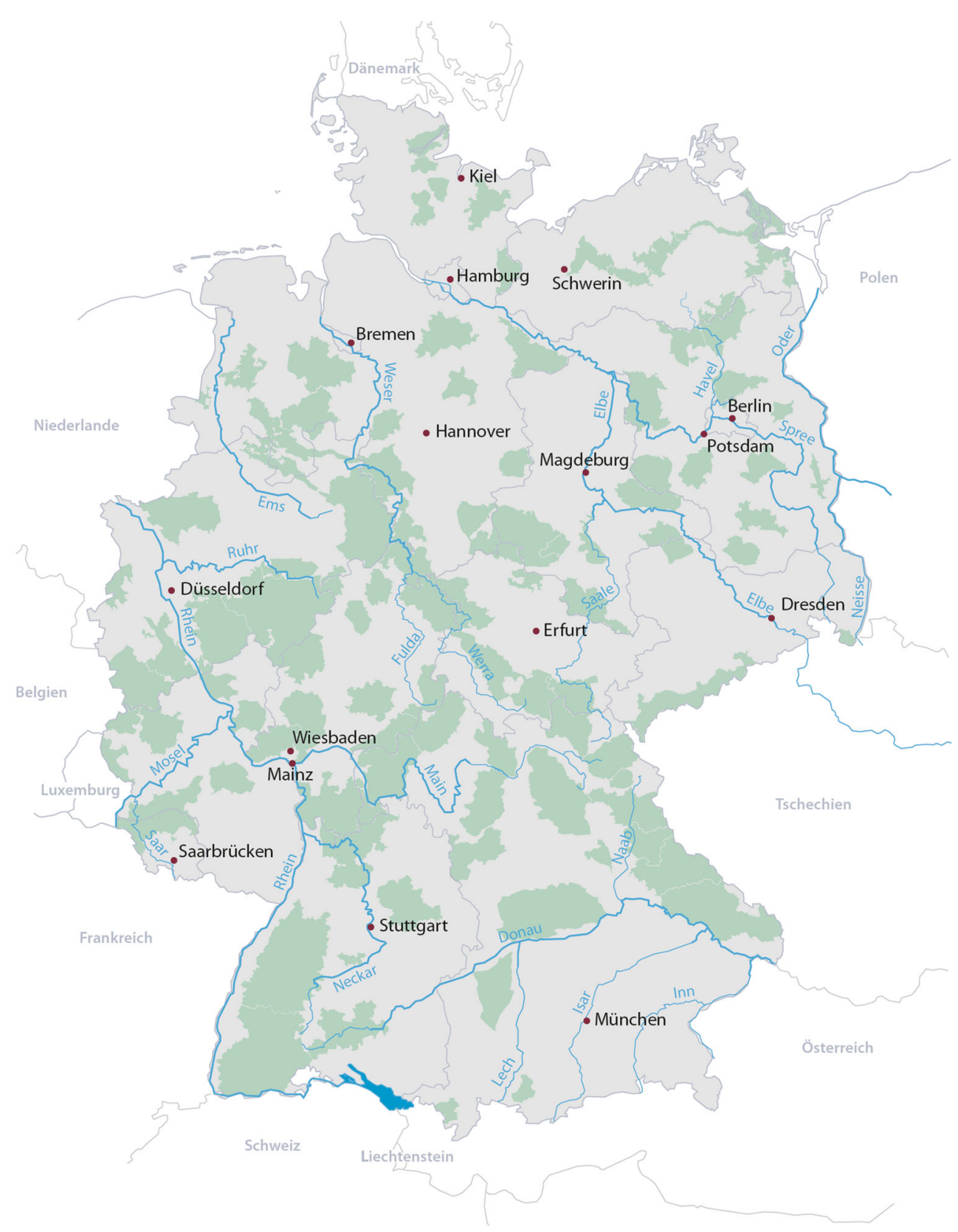

Abbildung 1 Die Naturparke in Deutschland (Stand 2021)

Quelle: Verband Deutscher Naturparke e.V.

lungsbedarfe und -möglichkeiten, zum Beispiel in Bezug auf die Lobbyarbeit, liegen können (Kapitel 3). Wir schließen mit einem resümierenden Fazit und einem Ausblick (Kapitel 4).

\section{Die Aufgabe der nachhaltigen Regionalentwicklung im Kontext der Regional-Governance-Debatte}

Naturparke befinden sich, wie in der Einleitung skizziert, im Spannungsfeld von Schutz- und Nutzengedanken. Wie beispielsweise Mose (2009: 11) betont, stellt der Gebietsschutz „das zentrale Instrument des modernen Naturschutzes“ dar, wozu die Naturparke flächenbezogen einen weitreichenden Beitrag leisten können. Aus der Historie heraus (vgl. aus- 
führlich Weber 2013) und in Verbindung mit dem heutigen Aufgabenportfolio nimmt zudem der Entwicklungsgedanke im Zusammenspiel von Mensch und Natur einen zentralen Stellenwert ein (Jedicke/Liesen 2017; Schöttle/Cammerer 2019; Weber/Weber 2019).

Eine nähere Verortung zur Kontextualisierung kann hier aus der theoretisch-konzeptionellen und gleichzeitig praktischen Diskussion um governance und speziell regional governance abgeleitet werden (vgl. Benz/Dose 2010; Böcher 2018; Berr/Jenal/Kühne et al. 2019): Ohne die Komplexität der Begrifflichkeit zu vernachlässigen, lässt sich der Terminus governance vor dem Hintergrund vorheriger politikwissenschaftlicher Steuerungsdiskussionen aus den 1980erJahren mit der Beobachtung assoziieren, dass ,im modernen Staat immer seltener die hierarchische Steuerung durch ein unitaristisches, staatlich übergeordnetes Steuerungszentrum zu beobachten ist, sondern dass vielmehr viele staatliche Ebenen und Akteure an der Produktion von Politiken beteiligt sind" (Böcher 2018: 58). Staatliches Handeln verändert sich in einer Weise, dass ,softe' Steuerungsformen an Bedeutung gewinnen, Akteurskonstellationen sich auffächern und „,netzwerkartige Regulierungsformen bis hin zu Formen privater Selbststeuerung “ etabliert werden (Bröchler/von Blumenthal 2006: 8f.). Statt Top-down-Umsetzung wird entsprechend von Bottom-up-Ansätzen ausgegangen, die sich im lokalen oder regionalen Kontext verorten lassen, womit die „subnationale, gemeindeübergreifende Ebene“ (Job/Woltering/Warner et al. 2016: 489) zu einem der Zugangspunkte veränderter Steuerung wird. Seit den 1990erJahren werden hiermit verbunden governance-Konzepte für regionalpolitische Entwicklungsprozesse herangezogen, die unter regional governance verhandelt werden, das heißt, die räumlich-mesoskalige Maßstabsebene wird konstitutiv (vgl. einführend Fürst 2001; Böcher 2006; Fürst 2010). In der Zusammenarbeit von öffentlichen Einrichtungen unterschiedlicher Ebenen und privat(wirtschaftlich)en Akteuren wird auf eine regionale Selbststeuerung gesetzt (Rhodes 1996; von Ruschkowski/Nienaber 2016; Nienaber 2018), um spezifische regionalpolitische Ziele zu erreichen, darunter unter anderem die ,nachhaltige Regionalentwicklung ' (Böcher 2018: 59). Kooperationen erfolgen vor dem Hintergrund gemeinsamer Frage- bzw. Problemstellung(en) im Idealfall über (temporär) vereinbarte Partnerschaften, auf funktionale Zielsetzungen hin ausgerichtet. Als Anreizsystem fungieren dabei z. B. Förderprogramme (Schubert 2004: 181; EUROPARC Deutschland 2013: 6; Böcher 2018: $60 \mathrm{f}$.). Das Bottom-up-Handeln wird als Vorteil angesehen, um die Akzeptanz von Maßnahmen zu erhöhen, die sich bei Verordnung von oben zunehmend einer Legitimation entziehen (Mose/Hammer/Siegrist et al. 2019: 84).

Eine so gedachte ,Regionalentwicklung ' wird nun dezidiert für Naturparke über deren „dynamisch-integrativen
Gebietsschutz", wie Weixlbaumer, Siegrist, Mose et al. (2015: 83) diesen betiteln, bzw. über eine dahingehende Gebietsentwicklung anschlussfähig. Letztlich lässt sich für den Gebietsschutz in Europa sogar insgesamt eine Ausweitung des Aufgabenportfolios über Naturschutz hinaus beobachten (Pütz/Job 2016; Mose/Hammer/Siegrist et al. 2019: 82). Schutz und Erholung als ,klassische“ Naturparkaufgabenfelder erfahren damit eine Aufweitung, um auch ,nachhaltige Nutzung' einer vertieften Bearbeitung im regionalen Kontext zuzuführen (Kaether 1994; Böcher 2018: 63; Forst 2019: 376) - basierend auf Ansätzen einer endogenen bzw. eigenständigen Regionalentwicklung seit den 1980er- bzw. 1990er-Jahren vor dem Hintergrund des prominent gewordenen Nachhaltigkeitsgedankens (Weber 2013: $71 \mathrm{ff}$; Mose/Hammer/Siegrist et al. 2019: 86). Regionalentwicklung wird häufig insbesondere auf sozioökonomische Veränderungen bezogen, ist gleichzeitig aber darüber hinausgehend zu verorten (Job/Woltering/Warner et al. 2016: 489; Majewski/Engelbauer/Job 2019), indem soziale und ökologische Komponenten einfließen - letztere explizit mit Bezug auf eine, nachhaltige Entwicklung . Dabei umfasst der Nachhaltigkeitsgedanke - in entkomplexisierender Kurzfassung - die Vorstellung, dass Ökonomie, Ökologie und Soziales als gleichberechtigt berücksichtigt und ausbalanciert miteinander verbunden werden, verknüpft mit den Zielen einer intra- und intergenerationellen Gerechtigkeit sowie ,Chancengleichheit" (Erdmann 1998; Kühne 2011: 296; Kühne/Meyer 2015). Mit Beschluss vom Herbst 2015 liegen nunmehr 17 Ziele nachhaltiger Entwicklung (SDGs, sustainable development goals) durch die Vereinten Nationen vor $^{3}$, die eine ,Nachhaltigkeitsagenda ' bis 2030 konturieren und so eine stärkere ,Nachhaltigkeits-Systematik' bieten, an der Handeln orientiert werden kann (Job/ Woltering/Warner et al. 2016: 482; Köster 2019: 436).

Vor diesem Hintergrund lässt sich die Verankerung der nachhaltigen Regionalentwicklung seit 2002 im Bundesnaturschutzgesetz als konsequent einordnen, um Naturparke als ,regionale Player' zu stärken, die Projekte anstoßen und koordinieren (Weber 2013; Weber/Weber 2015; Mehnen/ Kabelitz/Liesen 2018). Die Aufgabe ist zu einer zentralen Säule der Naturparkarbeit geworden (VDN 2018a: 4), entzieht sich allerdings einer ,einfachen Definition' und Umsetzung. So stellt sich die Frage, wie ,in der Praxis ' der Naturparkarbeit Aufgabenumriss und konkrete Bearbeitung ausgestaltet wurden und werden., Theorie und Praxis ' gehen dabei stellenweise durchaus auseinander, wie wir im Folgenden herausarbeiten - zunächst in einer resümierenden $\mathrm{Zu}$ sammenschau, gefolgt von der Gegenüberstellung quantita-

\footnotetext{
3 https://sdgs.un.org/goals (25.05.2021).
} 
tiver Befragungen der Naturpark-Geschäftsführungen/-verwaltungen 2011 und 2020.

\section{Nachhaltige Regionalentwicklung in Naturparken - Veränderungen im Zeitverlauf}

\subsection{Herausforderungen und Chancen - eine resümierende Zusammenschau}

Im Feld der Großschutzgebiete kommt Biosphärenreservaten originär die Rolle der ,Modellregionen' für nachhaltige Entwicklung zu (vgl. Borsdorf/Jungmeier/Braun et al. 2020). Ihre Auswahl resultiert aus dem Ziel, Gebiete zu schützen und zu entwickeln, die ,für bestimmte Landschaftstypen charakteristisch sind" ( $\$ 25$ Abs. 1 S. 1 BNatSchG), was deren Anzahl von derzeit 18 in Deutschland begründet. Naturparke mit ihrem allgemeineren Schutz- und Entwicklungsziel von Kulturlandschaften beanspruchen die Aufgabenerfüllung im Nachhaltigkeitsund Regionalentwicklungskontext nun ebenfalls für sich, verbunden mit dem Hinweis darauf, dass sie „Vorbilder für eine nachhaltige Regionalentwicklung“ und „Ideallandschaften" werden können (Weber/Weber 2015: 151) - forciert durch den Verband Deutscher Naturparke e. V. (VDN) als Dachverband (Liesen/Köster/Porzelt 2008; Köster 2019; VDN 2020). Dem VDN folgend sollen die über 100 Parke in Deutschland die „Rolle von Motoren und Moderatoren für die ländliche Regionalentwicklung wahrnehmen“ (VDN 2009: 14), was auf große Ambitionen schließen lässt und sich in einer Vielzahl an (potenziellen) Handlungsfeldern niederschlägt, die weit über Naturschutzaspekte hinausgehen und explizit ökonomische und soziale Belange umfassen: Hierzu zählen unter anderem der Aufbau von Kooperationen unter Einbindung der Bevölkerung, ein regionales Wirtschaften in Koppelung an eine nachhaltige Landnutzung und den Erhalt der Biodiversität, die Vermarktung regionaler Produkte, eine nachhaltige Siedlungsentwicklung unter Berücksichtigung regionaler Baukultur, die Vernetzung kultureller Angebote, die Stärkung regionaler Identität, der Aufbau von Naturpark-Partner-Netzwerken, die Förderung einer umweltverträglichen Mobilität, eine ,landschaftsverträgliche“ Nutzung erneuerbarer Energien und nachwachsender Rohstoffe sowie Maßnahmen zum Klimaschutz und zur Klimaanpassung (Köster 2019; VDN 2020; vgl. beispielhaft Abbildung 2). Auch im Rahmen der seit 2006 fortlaufenden ,Qualitätsoffensive Naturpar$\mathrm{ke}^{\prime}$ des VDN stellt die nachhaltige Regionalentwicklung einen elementaren Baustein dar. Die Qualitätsoffensive bildet für die Naturpark-Trägerorganisationen ein Instrument zur Selbsteinschätzung, zur Lenkung von Ressourcen und zur fortlaufenden Verbesserung der Naturparkarbeit. Sie eignet sich außerdem als Ausgangspunkt, fördernde und hemmende Faktoren zu erkennen sowie auf der Grundlage der Ergebnisse konkrete Empfehlungen für die künftige Naturparkarbeit abzuleiten (Liesen/Schäfer 2019).

Bisherige Untersuchungen zu den deutschen Naturparken zeigen im Hinblick auf die Aufgabenumsetzung sowohl verschiedene Probleme als auch Potenziale auf, die zur weiteren Verortung resümierend zusammengefasst werden. Auf Seiten der Herausforderungen sticht zunächst eine Begriffsunschärfe hervor, was denn genau unter ,nachhaltiger Regionalentwicklung ' zu verstehen sei - mitunter wird der Übergang zu anderen Aufgaben des Naturschutzes oder der nachhaltigen Regionalentwicklung als fließend bewertet (Weber 2013: $144 \mathrm{ff}$.). Als stark limitierende Faktoren werden begrenzte bzw. unzureichende finanzielle und personelle Ressourcen angesehen. Eigentlich setze die Aufgabenbandbreite eine starke Handlungsfähigkeit und strukturelle Grundlagen voraus, die aber nicht vollumfänglich gegeben seien (Weber/Weber 2015: 152f.; Gehrlein/Mathias/ Steil 2019: 390). Die Personalausstattung liege, nach Forst (2019: 380) und dem VDN (2017a, 2017b), im Vergleich zu Naturparken in anderen Ländern der Europäischen Union auf einem hinteren Rang. Immer wieder werden damit ressourcenbezogene Kapazitätsgrenzen erreicht, woraus etwa Restriktionen bei der Beantragung von Fördermitteln (Weber 2013: 193 ff.) oder der Erarbeitung von Naturparkplänen, wie im „Wartburger Programm“ (VDN 2018a) für die Naturparke 2030 angestrebt, resultierten (Liesen/Hoheisel/ Mengel et al. 2017). Im Bereich nachhaltiger touristischer Entwicklung fungierten die Parke zudem noch nicht weitreichend als Katalysatoren von entsprechenden Angeboten (Majewski/Engelbauer/Job 2019: 425), wobei es regional auch bereits innovative Ansätze gibt („Münstertaler Modell“, vgl. Liesen/Coch 2015), was allgemeiner die Frage nach der tatsächlichen Positionierung als ,wichtiger regionaler Akteur' im Zusammenspiel mit anderen Interessenvertretern aufwirft (Weber 2013: $207 \mathrm{ff}$.). Gerade im Bereich Tourismus ist allerdings zu berücksichtigen, dass es zahlreiche Stakeholder gibt, die die Aufgabe der touristischen Entwicklung erfolgreich wahrnehmen und somit Naturparke nur ein Baustein eines (touristischen) Netzwerkes sind (Risthaus/Schubert 2017). Auf der inhaltlichen Ebene werden Zielkonflikte unter anderem zwischen Naturschutz und Landnutzung gesehen (Gehrlein/Mathias/Steil 2019: 391).

Diesen Aspekten stehen mehrere Potenziale gegenüber: Eine große Zahl der Naturparke besteht seit mehreren Jahrzehnten - sie stellen eine ,tradierte Gebietskulisse ' dar und sind regional als beständige und zudem kommunale, die Grenzen von Landkreisen und/oder sogar von Bundesländern überschreitende Institutionen verankert (Weber/ Weber 2014; Weber/Weber 2015: 153). Eine Zusammen- 

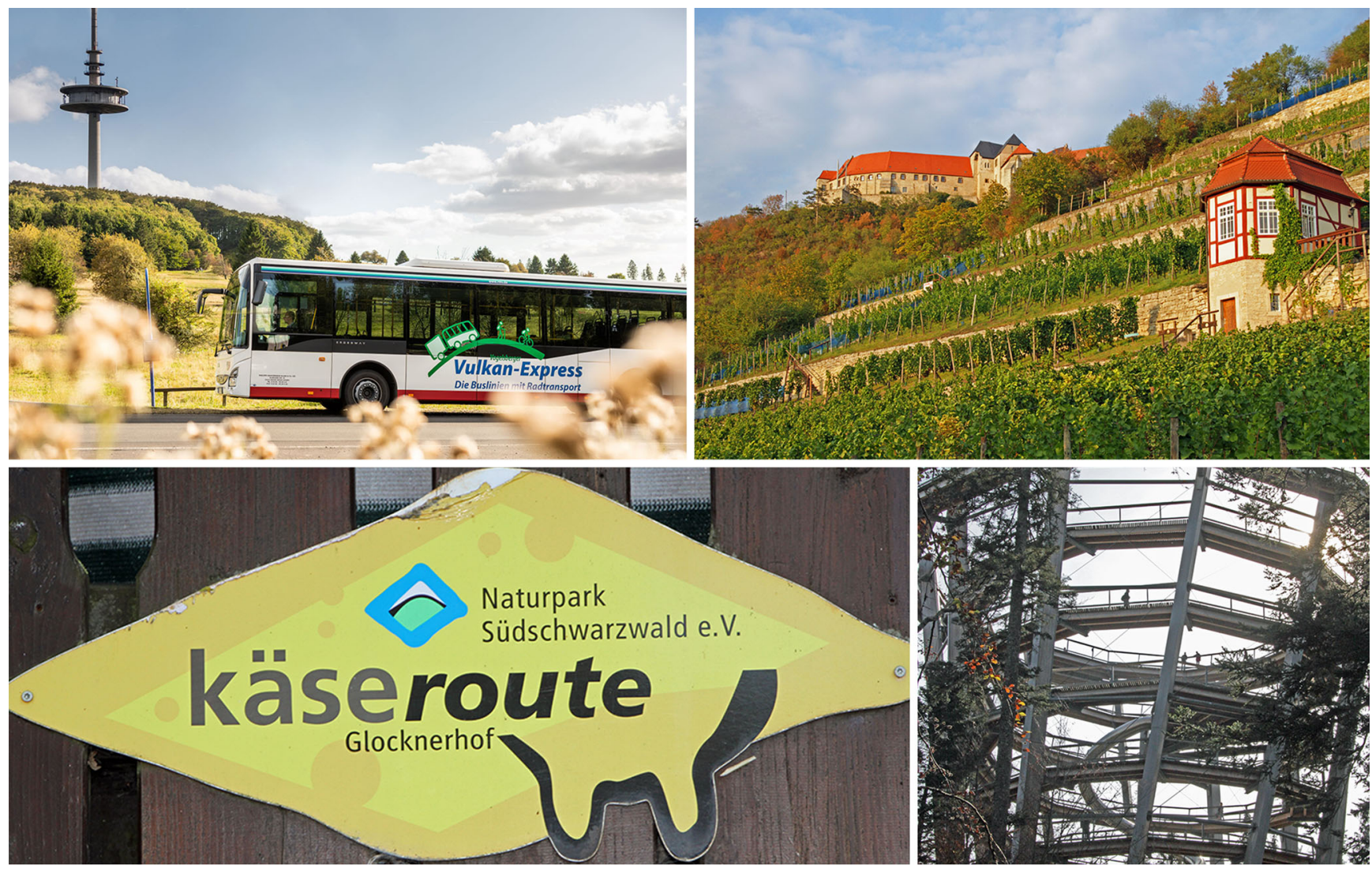

Abbildung 2 Ein Einblick in die Vielfalt der Aufgabenwahrnehmung nachhaltiger Regionalentwicklung: ÖPNV-Angebote im Naturpark Hoher Vogelsberg, Erhalt historischer Weinbau-Kulturlandschaften im Naturpark Saale-Unstrut-Triasland, Vermarktung regionaler Spezialitäten im Naturpark Südschwarzwald, Natur- und Landschaftserlebnis sowie Wissensvermittlung über einen Baumkronenpfad im Naturpark Schwarzwald Mitte/Nord

Quelle: VGO Steffen Löffler (links oben), alle anderen Aufnahmen Jörg Liesen

arbeit von Akteuren kann prinzipiell durch sie koordiniert werden (Mehnen/Mose/Strijker 2013; Weber/Weber 2015; Mayer/Stoll-Kleemann 2020: 484), um so verschiedene Belange im Feld nachhaltiger Regionalentwicklung - gerade in der interdisziplinären Verbindung aus Naturschutz und Entwicklung ländlicher Räume (Jedicke/Liesen 2017) zusammenzuführen, was gleichzeitig hinreichende (personelle) Ressourcen erforderlich macht (von Ruschkowski/ Nienaber 2016; Mehnen/Kabelitz/Liesen 2018). Zudem fungierten sie bereits durchaus und könnten auch stärker als Kooperationsplattform unter anderem für LEADER ${ }^{4}$ - und weitere Förderprogramme dienen (Gehrlein/Mathias/Steil 2019: 392f.). Aufgrund der Vielfalt an Aufgabenfeldern seien Naturparke darüber hinaus an viele gesellschaftliche Bereiche anschlussfähig, zu denen Beziehungen hergestellt

4 EU-Programm zur Förderung von innovativen Maßnahmen im ländlichen Raum (Liaison entre actions de développement de l'économie rurale). werden können, womit der ,integrative Charakter“ zur Stärke gerieren kann (Köster 2019: 437). Schöttle und Cammerer (2019: 420) gehen - um hieran anzuschließen - davon aus, dass die Parke immer mehr zu ,wichtige[n] Impulsgeber[n], Netzwerker[n] und Umsetzer[n] für die nachhaltige Regionalentwicklung" werden.

Naturparke könnten - wenn die Potenziale gegenüber den Herausforderungen überwiegen - perspektivisch immer stärker, wie es Weber (2013) bereits als Chance formulierte, zu ,Managern' der nachhaltigen Regionalentwicklung werden, um so auch eine Profilierung in mitunter vorhandener Konkurrenz zu den anderen Großschutzgebieten zu erlangen (vgl. Liesen/Weber 2018; Weber/Weber 2019). In idealtypischer Betrachtung schreiben sich Naturparke somit in die Regional-governance-Debatte ein - zur vertiefenden Analyse bedarf es wiederum weitergehender Forschungsarbeiten (vgl. Köster 2019: 437). 


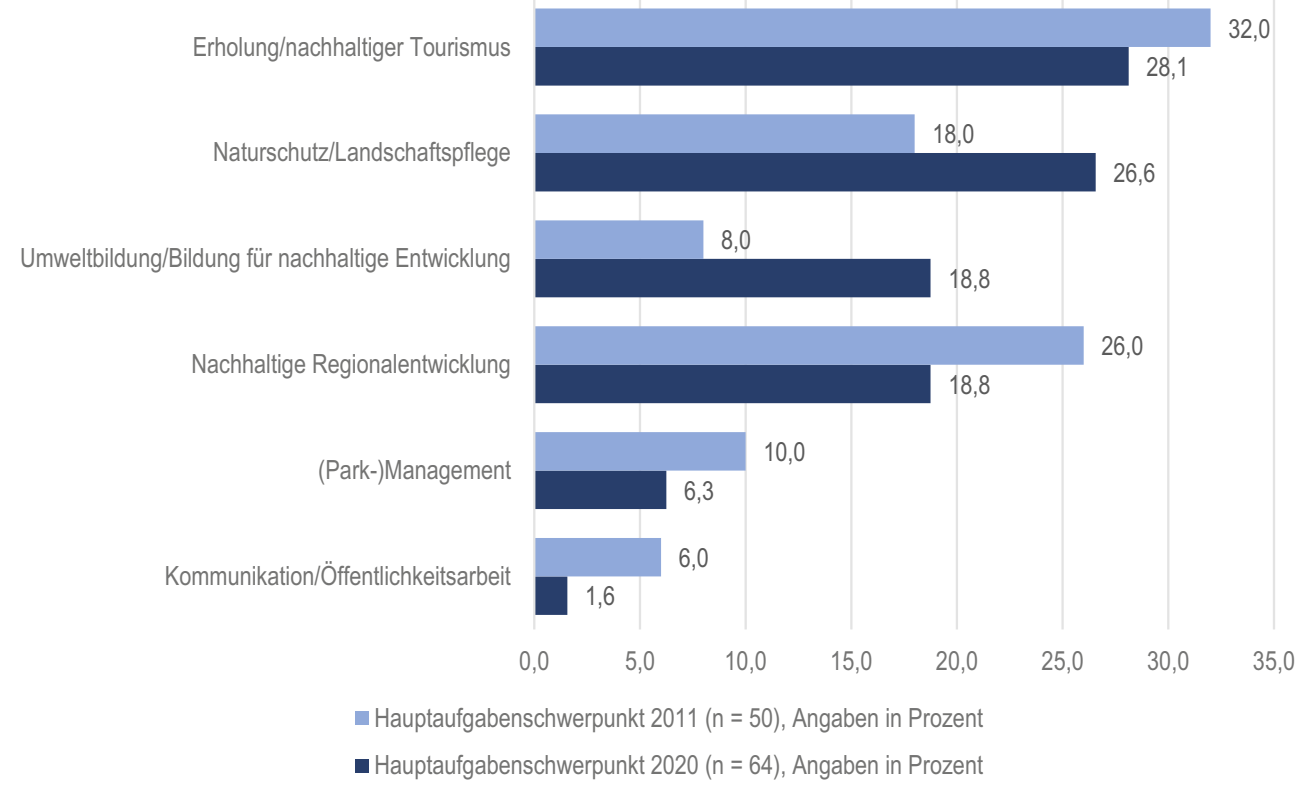

Abbildung 3 Hauptaufgabenschwerpunkte der Naturparke

Quelle: Eigene Darstellung, Datengrundlage Erhebung 2011 (vgl. Weber 2013) und eigene Erhebung 2020

\subsection{Zentrale Ergebnisse der vergleichenden Betrachtung 2011 und 2020}

Die Zusammenschau von Herausforderungen und Chancen von Naturparken im Hinblick auf die Aufgabenumsetzung der nachhaltigen Regionalentwicklung konnte einen Überblick geben, hat allerdings nicht dem Faktor Zeit aktiv Rechnung getragen: Welche Veränderungen lassen sich im Zeitverlauf beobachten? Hierzu kann ein Beitrag geleistet werden, indem wir Ergebnisse zweier quantitativer Befragungen aus den Jahren 2011 und 2020 in Beziehung setzen. Weber (2013) hat im Sommer 2011 alle damals bestehenden 104 Naturparke - konkret deren Geschäftsführer/-innen - angeschrieben und um postalische Beantwortung eines Fragesets aus geschlossenen und offenen Fragen zur Aufgabe der nachhaltigen Regionalentwicklung gebeten. 56 Parke nahmen damals an der Umfrage teil, was einer sehr guten Ausschöpfung von 53,8 \% entsprach. Im Herbst 2020 wurde die Umfrage mit einem reduzierten Frageset wiederholt, wobei dieses Mal eine Ansprache per E-Mail durch den VDN erfolgte und das Ausfüllen des Fragebogens über ein Online-Befragungstool ermöglicht wurde. 64 der 101 angeschriebenen Parke (101 Mitglieder beim Dachverband VDN) beteiligten sich, das heißt 63,4\%. Insbesondere Naturparke, die in den 1960er-, 1990er- und 2000er-Jahren gegründet wurden, nahmen an den Befragungen teil; das entspricht den zentralen Gründungsphasen. Innerhalb der Gründungsjahrzehnte bestehen zwischen 2011 und 2020 keine markanten Unterschiede, was, von diesem Kriterium ausgehend, eine gute Vergleichbarkeit ermöglicht. Zentra- le Verschiebungen, aber auch fortbestehende Bewertungen rücken nun in den Fokus.

Um dem propagierten Ziel der ,Modellregionen für nachhaltige Entwicklung“ (VDN 2018a: 4) gerecht zu werden, wird bei den Naturparken auf eine Verbindung aus Schutz, Erholung, Bildung und Entwicklung gesetzt (vgl. Kapitel 3.1), was verbunden mit dem (Park-)Management sowie Kommunikation und Öffentlichkeitsarbeit zentrale Aufgabenfelder konturiert. Im Hinblick auf eine Gewichtung dominiert sowohl $2011\left(\mathrm{n}=50^{5}\right)$ als auch $2020(\mathrm{n}=$ 64) Erholung/nachhaltiger Tourismus (2011: 32,0\% bzw. 2020: 28,1\%). Einen erheblichen Bedeutungsgewinn haben 2020 Naturschutz/Landschaftspflege (Anstieg von 18,0\% auf 26,6\%) und Umweltbildung/Bildung für nachhaltige Entwicklung (von 8,0\% auf 18,8\%) erfahren. Ein ,klassisches“ Aufgabenfeld der Naturparkarbeit erlebt so einen neuen ,Schub“, was mit Diskussionen und Engagement um biologische Vielfalt, Klimawandel, Wildnis und Natura2000-Management in Verbindung gebracht werden kann (vgl. Jedicke/Liesen 2017; VDN 2018b; Brackhane/Liesen/ Bieber et al. 2019). Bildung für nachhaltige Entwicklung

\footnotetext{
5 Zwar haben 2011 insgesamt 56 und 2020 insgesamt 64 Naturparke an den Befragungen teilgenommen, aber nicht alle Fragen wurden durchgehend beantwortet. Mit den n-Angaben wird daher kenntlich gemacht, wie viele Geschäftsführungen auf die jeweiligen Fragen geantwortet haben. Es sei zudem angemerkt, dass zum besseren Lesefluss von „den Naturpark-Geschäftsführungen“ gesprochen wird, sich die Angaben aber automatisch auf diejenigen beziehen, die an den Umfragen teilgenommen haben.
} 


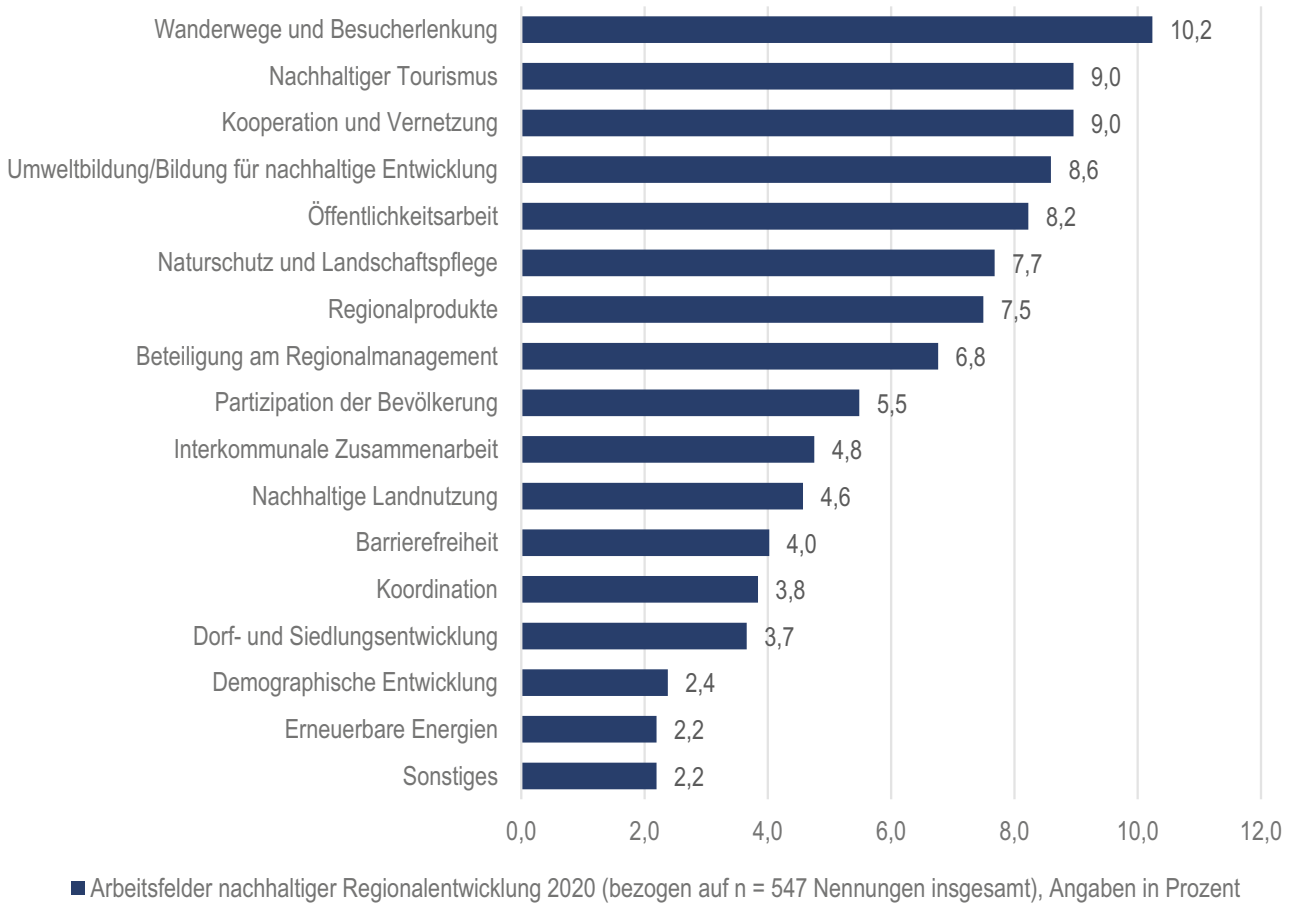

Abbildung 4 Wichtige Arbeitsfelder der nachhaltigen Regionalentwicklung in Naturparken (2020) Quelle: Eigene Darstellung, Datengrundlage eigene Erhebung 2020

ist seit 2017 im Bundesnaturschutzgesetz für Naturparke (§ 27 Abs. 2 BNatSchG) verankert (Forst 2019: 374), sodass der Bildungsaspekt eine Stärkung erfahren hat (vgl. auch Horstick/Schubert 2019) - unterstïtzt und forciert durch das „Wartburger Programm“, das Naturparke als „Bildungsregionen für nachhaltige Entwicklung“ (VDN 2018a: 2) ausruft. Erst an vierter Stelle folgt nachhaltige Regionalentwicklung, bei der ein Rückgang von $26,0 \%$ auf $18,8 \%$ zu verzeichnen ist (vgl. Abbildung 3).

Hat also die Aufgabe der nachhaltigen Regionalentwicklung in den letzten zehn Jahren an Relevanz eingebüßt? Die provokante Frageformulierung deutet bereits darauf hin, dass die Antwort differenziert ausfallen muss. Bereits 2011 $(\mathrm{n}=54)$ gaben $70,4 \%$ der Geschäftsführungen an, nachhaltige Regionalentwicklung umzusetzen, 2020 ( $\mathrm{n}=63$ ) sind es $82,5 \%$. Nur 6,3\% wollen der Aufgabe auch weiterhin keine Aufmerksamkeit schenken - 2011 waren es noch 14,8\%. ,Nachhaltige Regionalentwicklung' ist also fest im Bewusstsein der Naturpark-Geschäftsführungen verankert. Hinzu kommt, dass verschiedene Aufgaben, die sie 2011 und weiterhin auch 2020 - unter Regionalentwicklung subsumieren, formal betrachtet nach dem VDN eigene Aufgabenfelder darstellen: nachhaltiger Tourismus, Naturschutz und Landschaftspflege, Bildung für nachhaltige Entwicklung, aber auch Öffentlichkeitsarbeit (vgl. Abbildung 4). Damals wie heute lassen sich die Arbeitsfelder aus der Perspektive der Naturparke so nicht genau trennen. 2020 werden Wanderwegemanagement und Besucherlenkung $(10,2 \%)$ als besonders wichtig angesehen, gefolgt von nachhaltigem Tourismus sowie Kooperationen und Vernetzungen (jeweils 9,0\%), Umweltbildung/Bildung für nachhaltige Entwicklung $(8,6 \%)$, Öffentlichkeitsarbeit $(8,2 \%)$ sowie Naturschutz und Landschaftspflege $(7,7 \%)$. Nachhaltige Regionalentwicklung bleibt eine Aufgabe mit einer großen Reichweite und Facettenvielfalt, wie durch Weber (2013) herausgearbeitet.

Während 2011 die Lage der Naturparke bezüglich Finanzen $(\mathrm{n}=56)$ und Personal $(\mathrm{n}=55)$ durchaus als sehr angespannt bezeichnet werden kann (Weber 2013: $173 \mathrm{ff}$.; Weber/Weber 2015: $152 \mathrm{f}$.), fallen die Bewertungen der Geschäftsleitungen 2020 erheblich positiver aus. 58,0\% stimmen jüngst der Aussage, die finanzielle Situation $(n=62)$ in ihrem Naturpark habe sich in den letzten Jahren verbessert, voll und ganz bzw. eher zu. 2011 waren es nur 19,7\%. Sehr ähnlich sieht es bei der vergleichbaren Frage im Hinblick auf die personelle Situation aus: Während 2011 nur 29,1 \% der Aussage einer Verbesserung voll und ganz bzw. eher zustimmten, sind es $2020(\mathrm{n}=62)$ insgesamt 56,5\% (vgl. Abbildung 5). Diese Einschätzungen spiegeln sich auch angesichts der konkreten Personalsituation wider: 71,9\% der Geschäftsführer/-innen nehmen $2020(\mathrm{n}=64)$ ihre Geschäftsführungsaufgabe in Vollzeit wahr, weitere 6,3\% mit 
Verbesserung der finanziellen Situation in den letzten Jahren

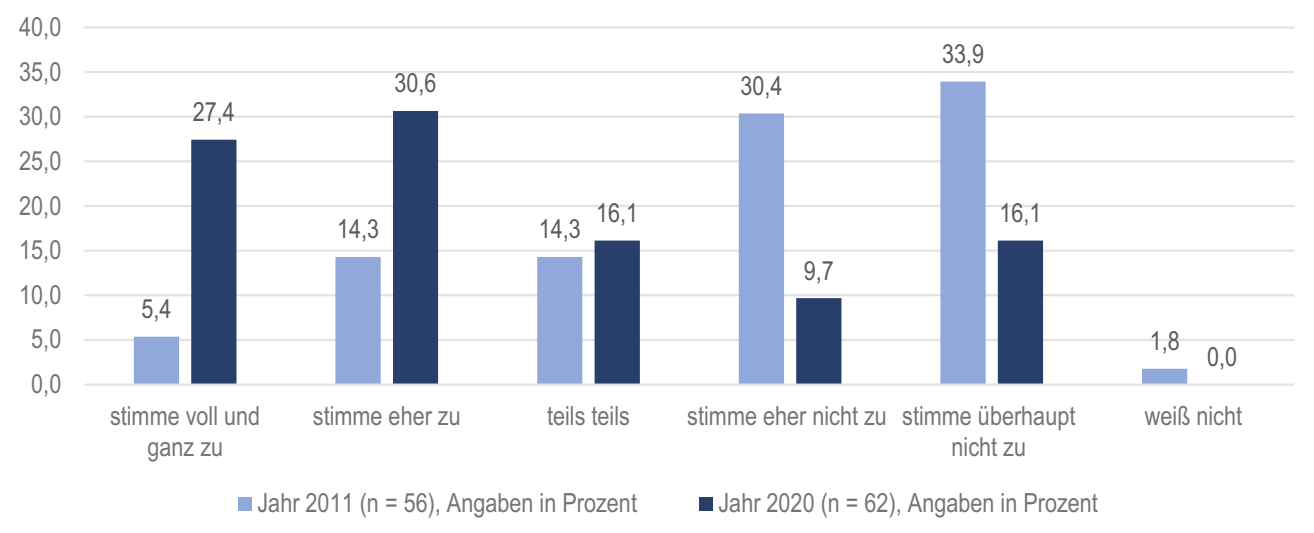

Verbesserung der personellen Situation in den letzten Jahren

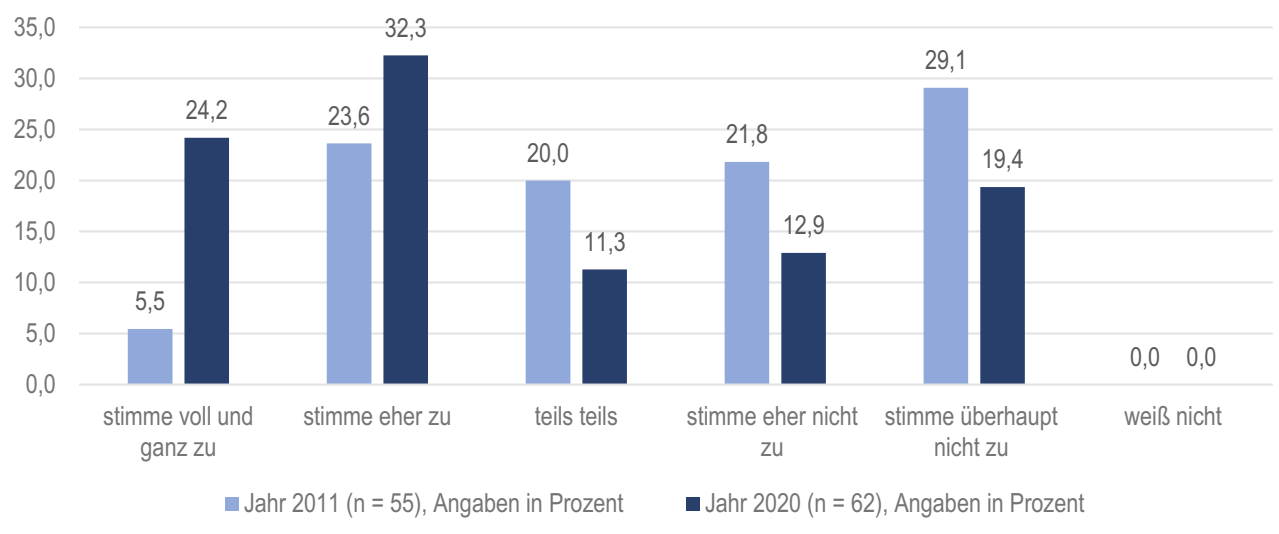

Abbildung 5 Bewertungen dahingehend, dass sich in den letzten Jahren die finanzielle (oben) bzw. personelle (unten) Situation der Naturparke verbessert habe Quelle: Eigene Darstellung, Datengrundlage Erhebung 2011 (vgl. Weber 2013) und eigene Erhebung 2020

mehr als 75 Prozent - 2011 waren es $63,6 \%$ bzw. 3,6\% $(\mathrm{n}=55)$, sodass eine Aufwertung der Leitungsaufgabe $\mathrm{zu}$ verzeichnen ist. Besonders markant fällt der Zuwachs der durchschnittlichen Beschäftigtenzahl aus, die sich von 1,8 $(\mathrm{n}=55)$ auf 4,5 $(\mathrm{n}=64)$ mehr als verdoppelt hat. Damit liegt sie auf dem Niveau der insgesamt als recht gut ausgestattet geltenden baden-württembergischen Naturparke und dem dortigen durchschnittlichen Personalbestand von 4,5 Arbeitskräften (Gehrlein/Mathias/Steil 2019: 391). Sie nähert sich so auch der Forderung nach sechs Vollzeitstellen für 2030 im „Wartburger Programm“ an (VDN 2018a: 3). Gleichzeitig erachten im Jahr 2020 (jeweils $\mathrm{n}=64$ ) die Naturpark-Geschäftsführungen - selbst wenn sie weniger kritisch als 2011 (jeweils $n=56$ ) antworten - sowohl die finanzielle als auch die personelle Situation weiterhin als ausbaufähig: In finanzieller Hinsicht halten sich die Zustimmung bzw. eine neutrale Position (zusammen 50,0\%; 2011: $32,1 \%)$ gegenüber der Ablehnung (50,0\%; 2011: 67,9\%) der Bewertung der aktuellen Lage als ausgezeichnet die
Waage. Die Personalsituation wird von knapp zwei Dritteln eher nicht oder überhaupt nicht (zusammen 65,7\%; 2011: $75,0 \%$ ) als ausgezeichnet eingeschätzt. Explizit wirkt sich die wahrgenommene Ressourcenknappheit auf die Umsetzung der Aufgabe der nachhaltigen Regionalentwicklung aus, die mit einer umfänglicheren Finanz- und Personalsituation besser ausfallen könnte, so die Geschäftsführer/-innen (basierend auf einer offenen Frage zu den Hemmnissen der Aufgabenerfüllung, $\mathrm{n}=$ je 54 Antworten 2011 und 2020; personell rund ein Drittel der Antworten, finanziell je etwa ein Fünftel mit leicht rückläufiger Tendenz im Vergleich).

Woher könnte nun - bei allem fortbestehenden Verbesserungsbedarf - die gewisse verbesserte Ausgangslage finanziell wie personell herrühren? Eine Analyse der Finanzierungsquelle lässt Rückschlüsse zu (2011: $\mathrm{n}=50,2020$ : $\mathrm{n}=$ 61). Weiterhin dominant fallen Landschaftspflege- bzw. Naturparkförderrichtlinien der Bundesländer aus (minimaler Anstieg von 66,0\% auf 68,9\% der Parke, die auf diese Mittel zurückgreifen), gefolgt von LEADER-Mitteln (60,0\% 


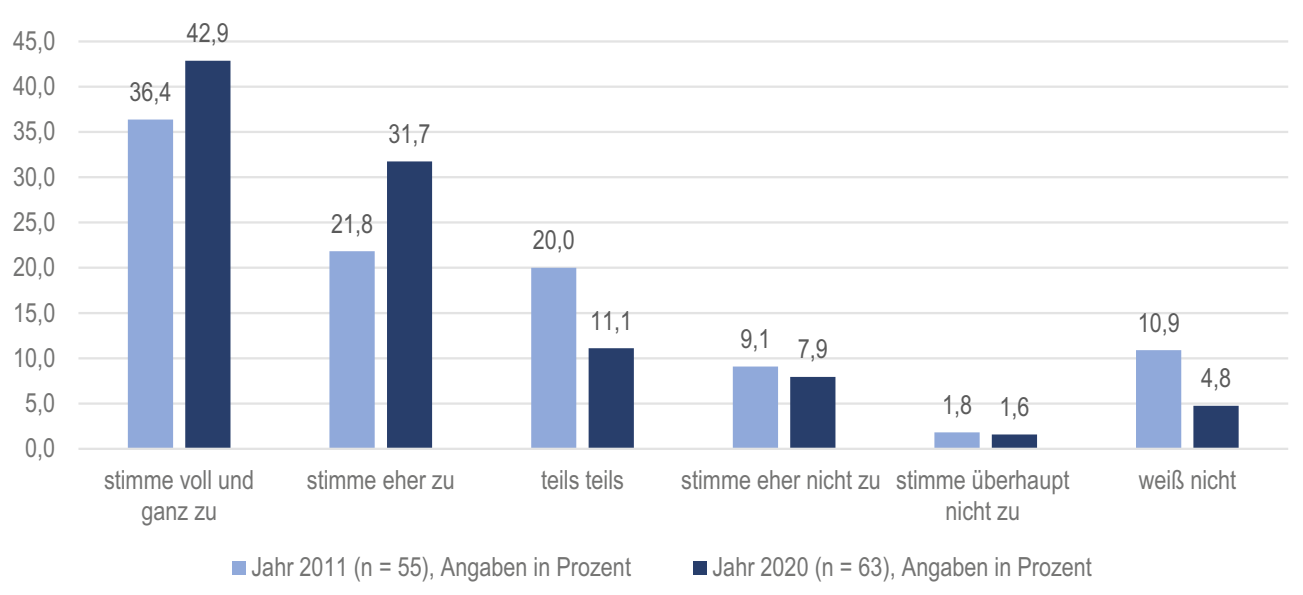

Abbildung 6 Zustimmung zur Aussage, dass es hilfreich für die Naturparkarbeit sei, dass die Aufgabe der nachhaltigen Regionalentwicklung in das Bundesnaturschutzgesetz aufgenommen wurde Quelle: Eigene Darstellung, Datengrundlage Erhebung 2011 (vgl. Weber 2013) und eigene Erhebung 2020

auf $67,2 \%$ ). Gerade die Förderprogramme der Länder haben markant an Bedeutung gewonnen: Gegenüber 28,0\% im Jahr 2011 konnten 2020 49,2\% der Parke von diesen profitieren. Während Interreg ${ }^{6}(16,0 \%$ auf $13,1 \%)$, LIFE $^{7}$ $(8,0 \%$ auf $6,6 \%)$ und weitere Programme der Europäischen Union $(38,0 \%$ auf $27,9 \%)$ etwas weniger genutzt werden, haben Förderprogramme des Bundes an Einfluss gewonnen $(10,0 \%$ auf $18,0 \%)$. Sponsoren werden immer wichtiger $(22,0 \%$ auf $34,4 \%)$. Dezidiert nach Letzteren gefragt (2011: $n=56,2020: n=64)$ ist auffällig, wie stark Privatpersonen Naturparke unterstützen: knapp ein Drittel der Parke $(31,3 \%)$, die an der Befragung 2020 teilnahmen, erhalten entsprechende Zuwendungen - 2011 war es noch nicht jeder zehnte Park $(7,1 \%)$. Ergänzend sind Sponsoren aus dem Energiesektor sehr viel stärker präsent: Während 2011 nur 3,6 \% der Parke Erträge aus diesem Bereich generieren konnten, sind es knapp zehn Jahre später 17,2\%. Nachhaltige Regionalentwicklung ermöglicht aus der Sicht der Naturpark-Geschäftsleitungen eine vielfältige Anschlussfähigkeit an Förderinstrumente aus unterschiedlichen öffentlichen wie privatwirtschaftlichen Bereichen, was sich gegenüber 2011 auch 2020 fortsetzt, in Teilen sogar noch einmal verstärkt.

Welche Bedeutung der Aufgabe der nachhaltigen Regionalentwicklung zugebilligt wird, zeigt sich, wenn sie in den Kontext der gesetzlichen Verankerung gestellt wird (2011:

\footnotetext{
6 https://www.interreg.de (17.05.2021).

7 LIFE (L'Instrument Financier pour l'Environnement) ist das einzige EU-Förderprogramm, das ausschließlich Umweltschutzbelange unterstützt (https://www.bmu.de/ themen/europa-internationales-nachhaltigkeit-digitalisierung/ europa-und-umwelt/life/ (17.05.2021)).
}

$\mathrm{n}=55,2020: \mathrm{n}=63)$. Knapp drei Viertel $(74,6 \%)$ der Geschäftsführer/-innen, die 2020 an der Befragung teilgenommen haben, geben an, dass es hilfreich sei (voll und ganz bzw. eher Zustimmung), dass die Aufgabe eine gesetzliche Verankerung erhalten habe $-16,4 \%$ mehr als 2011 (vgl. Abbildung 6). In der Rückschau scheint etwa zwanzig Jahre nach der Aufnahme in das Bundesnaturschutzgesetz die Tragweite und Relevanz noch deutlicher geworden zu sein. Die Potenziale liegen aus der Sicht des Parkmanagements 2011 wie 2020 zuvorderst in einer Bedeutungssteigerung und Imageverbesserung für die Naturparke (12,7\% im Jahr 2020, ausgehend von $\mathrm{n}=385$ Nennungen insgesamt bei offener Fragestellung), gefolgt von Chancen für die regionale Wertschöpfung und einer Stärkung des Regionalbewusstseins (je 11,7\%). Naturparke würden durch die Aufgabenverankerung auch stärker als Manager und Vernetzungsplattform $(9,9 \%)$ bzw. regionaler Player $(9,6 \%)$ wahrgenommen. So könnten weitere der grundlegend definierten Aufgaben eine bessere Bearbeitung erfahren. Eine entsprechende Wahrnehmung seitens des Managements lässt sich aufgrund der Bewertung mehrerer Aussagen unterstreichen: Während sich der Bekanntheitsgrad bei der einheimischen Bevölkerung $2011(\mathrm{n}=55)$ und $2020(\mathrm{n}=64)$ vergleichbar verhält (Anstieg von „stimme eher zu“ 23,6\% auf 29,7\%, dafür ebenfalls Anstieg von "stimme eher nicht zu“ von $20,0 \%$ auf 23,4\%), werden Naturparke bei regionalen Entscheidungsgremien (2011: $\mathrm{n}=56,2020: \mathrm{n}=64)$ stärker als wichtige regionale Akteure angesehen (Rückgang bei „stimme eher nicht zu“ von $26,8 \%$ auf $10,9 \%$ mit nahezu entsprechendem Zuwachs bei „stimme voll und ganz zu“ bzw. „stimme eher zu“) und sie könnten praktisch betrachtet regionale Entwicklung stärker mitbestimmen (Anstieg der Zustimmung bei voll und ganz bzw. eher um 6,3\% auf 


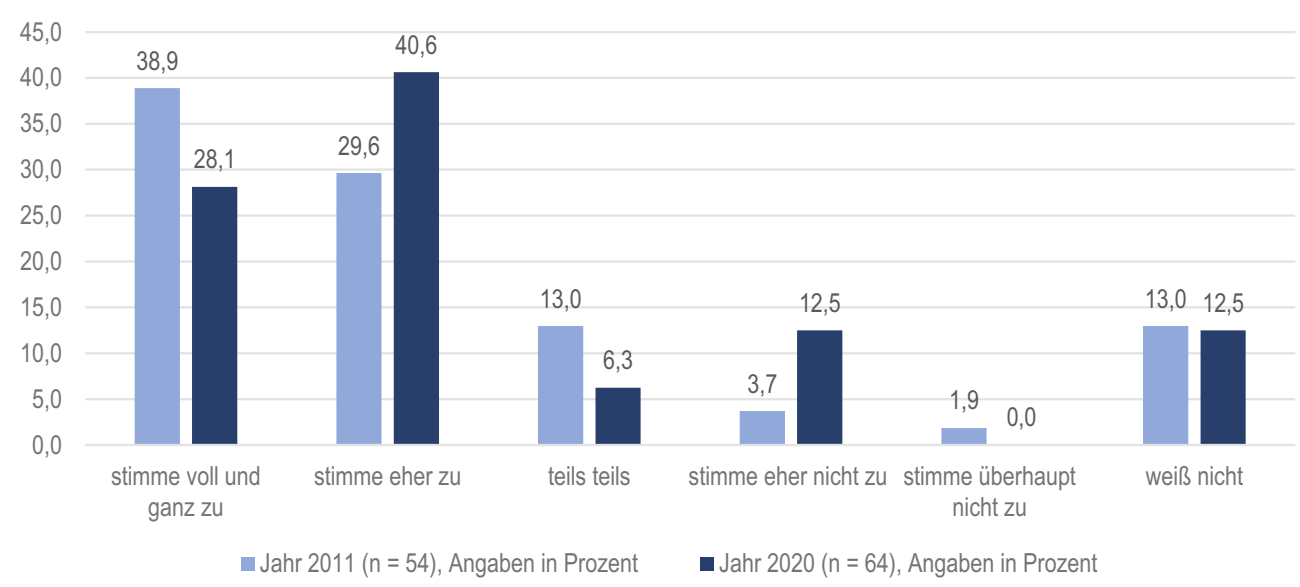

Abbildung 7 Zustimmung zur Aussage, dass Naturparke im Vergleich zu Nationalparken als Schutzgebiete zweiter Klasse behandelt würden

Quelle: Eigene Darstellung, Datengrundlage Erhebung 2011 (vgl. Weber 2013) und eigene Erhebung 2020

43,8\%, während die Ablehnung um 3,8\% rückläufig nur noch bei $14,1 \%$ liegt).

Die Vielfalt an Partnern, mit denen die Naturparke nach eigenen Angaben im Bereich der nachhaltigen Regionalentwicklung kooperieren, dürfte einen wichtigen Anteil an der regionalen Wahrnehmung haben: Gebietskörperschaften unterschiedlicher administrativer Ebenen, regionale Managements, Institutionen aus Tourismus, Forstwirtschaft, Naturschutz, Landwirtschaft, Privatwirtschaft. Gravierende Unterschiede zwischen $2011(\mathrm{n}=56)$ und $2020(\mathrm{n}=62)$ ergeben sich dabei nicht, abgesehen von wenigen ,Ausreißern': Kommunen sind relevanter geworden $(85,5 \%$ gegenüber $69,6 \%$ ), ebenso regionale Vermarktungsinitiativen $(53,2 \%$ gegenüber 39,3\%). Dafür wurde die Regionalplanung deutlich seltener genannt $(25,8 \%$ gegenüber $44,6 \%)$. Viele Akteursnetzwerke, regionaler Steuerung ' scheinen damit in der Gesamtbetrachtung eine Verankerung erfahren zu haben.

Gleichzeitig deuten sich potenzielle Überlappungen im Aufgabenfeld der nachhaltigen Regionalentwicklung an (2011: $\mathrm{n}=56,2020: \mathrm{n}=61$ ): Auch weitere Institutionen und Organisationen wie LEADER-Managements $(82,1 \%$ 2011 vs. 85,2\% 2020), Verbände, Stiftungen und Vereine (14,3\% vs. $73,8 \%$ ), Regionalmanagements der Landkreise $(64,3 \%$ vs. $70,5 \%)$ oder Wirtschaftsförderungen $(48,2 \%$ vs. 59,0\%) setzen aus der Sicht der Geschäftsführer/-innen Projekte im Bereich der nachhaltigen Regionalentwicklung um - und dies gegenüber 2011 in teils deutlich gewachsenem Ausmaß. Wahrgenommene Konkurrenzen und Kompetenzüberschneidungen nehmen zwar bei den Bewertungen keinen zentralen Raum ein, allerdings ist der Anstieg doch auffällig - von 7,4\% zu 13,0\% (ausgehend von einer offen gestellten Frage nach Hemmnissen bei der Aufgabenumsetzung, 2011 und 2020 jeweils $\mathrm{n}=54$ Antworten insgesamt).
Im Hinblick auf Entwicklungsperspektiven stellt sich für Naturparke im politischen Kontext auch die Frage nach ihrem ,Standing': Trotz zunehmender Bedeutung in der Regionalentwicklung und Wertschätzung durch regionale Entscheidungsstellen sehen sich die Naturparke im Verhältnis zu Nationalparken noch nicht auf, gleicher Augenhöhe' (vgl. weiterführend Chilla/Kühne/Neufeld 2016: $227 \mathrm{ff}$;; Jedicke/Liesen 2017; Liesen/Weber 2018) - eine Verbesserung gegenüber 2011 wird gleichzeitig 2020 konstatiert (vgl. Abbildung 7: die Aussage, Naturparke würden gegenüber Nationalparken als Schutzgebiete zweiter Klasse behandelt, wird weniger deutlich bejaht), sodass die weitere perspektivische Entwicklung genauer zu beobachten bleibt.

\subsection{Diskussion der Ergebnisse: Die gesetzliche Verankerung der nachhaltigen Regionalentwicklung als gelungener Schritt}

Wir diskutieren im Folgenden zentrale Ergebnisse und ordnen diese in den Gesamtzusammenhang des Beitrags ein, zur Illustration verknüpft mit Beispielen aus der Naturparkarbeit. Während Weber (2013) aufgrund der quantitativen Befragung der Naturparke im Jahr 2011 und vertiefenden Gesprächen mit unterschiedlichen Akteuren feststellte, dass damals noch keine Einigkeit über Inhalt und Spektrum der nachhaltigen Regionalentwicklung auf Umsetzungsebene existierte, haben die Naturparke durch ihre Arbeit in den letzten Jahren immer mehr Fakten geschaffen. Eine gewisse 2011 konstatierte Skepsis gegenüber der seit 2002 im Bundesnaturschutzgesetz verankerten Aufgabe ist im Jahr 2020 zurückgegangen. Es wird vielmehr in hohem Maße seitens der Geschäftsführer/-innen angegeben, die Aufgabe sei in ihrer gesetzlichen Verankerung ein Gewinn und 
umzusetzen (vgl. Abbildung 6). Gleichzeitig zeigt sich, dass weiterhin keine wirkliche Trennschärfe zu anderen Aufgabenbereichen der Naturparke besteht bzw. die nachhaltige Regionalentwicklung vielleicht eher als ,Dach“ oder Ankerpunkt für die Aufgabenvielfalt dient (im Abgleich aus Abbildung 3 und Abbildung 4 ersichtlich). Beispielhaft für eine netzwerkartige, regionale und nachhaltig definierte Steuerung einer ganzen Region sei hier der Naturpark Südschwarzwald genannt, der mit dem Naturpark-Plan 2025 (Naturpark Südschwarzwald 2018) den „Wegweiser für die nachhaltige Entwicklung der Naturparkregion“" (Schöttle/Cammerer 2019: 417) formuliert hat. Darin werden Aufgaben und Projekte definiert, die teilweise bereits seit mehreren Jahren etabliert sind und fortgeführt werden sollen, wie zum Beispiel Naturpark-Märkte oder die Vermarktung von Bio-Weiderind, beziehungsweise solche, die den Auftakt zu langfristigen Projekten markieren, wie der Erhalt regionaler Baukultur im Schwarzwald oder die Entwicklung von Klimaanpassungsstrategien mit und für die Landwirtschaft und den Naturschutz. Qualifizierte und aktuelle Naturparkpläne werden für solche Projektideen zu einem zentralen Orientierungspunkt, der auch eine gewisse Systematik in der Tätigkeitsbandbreite ermöglichen kann (Liesen/Hoheisel/Mengel et al. 2017; Gehrlein/Mathias/ Steil 2019; VDN 2019).

Um in einem letztlich hochgradig komplexen Bereich tätig zu sein, bedarf es hinreichender Ressourcen. In vielen Naturparken hat sich für diese erfreulicherweise in den letzten zehn Jahren sowohl die Finanz- als auch die Personalsituation verbessert (vgl. Abbildung 5), unter anderem durch eine umfangreichere Landesförderung (vgl. weiterführend Liesen/Schäfer 2019). Auch Förderprogramme wie LEADER oder der Zugriff auf Bundesmittel sind Möglichkeiten der (Projekt-)Finanzierung, die verstärkt genutzt werden. Die Akquise dieser Mittel hängt eng mit der verbesserten Personalsituation zusammen, denn ohne qualifiziertes Personal kann nur eine begrenzte Beantragung von Fördermitteln und keine qualifizierte Projektbearbeitung oder auch keine ausreichende, impulsgebende Beteiligung in LEADER-Regionen erfolgen (vgl. Gehrlein/Mathias/ Steil 2019). Damit hat sich zumindest eines der von Weber (2013: $173 \mathrm{ff}$.) konstatierten Problemfelder verbessert, erfüllt aber noch nicht die Forderungen der Naturparke mit ihrem Dachverband VDN, dass in jeder Geschäftsstelle sechs Vollzeitkräfte alle gesetzlich definierten Handlungsfelder (Naturschutz und Landschaftspflege, nachhaltige Regionalentwicklung, nachhaltiger Tourismus, Bildung für nachhaltige Entwicklung) abdecken sollen (VDN 2018a: 3; Köster 2019: 437). Die Erfüllung von Aufgaben in den Bereichen Schutz, Erholung, Bildung und Entwicklung (VDN 2018a: 4) bedingen durch die Vielfalt und Komplexität bereits einen ,soliden“ Personalstamm, der - eng verbun- den - stark gefordert ist, wenn Förderprojekte akquiriert und umgesetzt werden sollen. Selbst wenn diese Anforderungen durchgehend erreicht wären, bliebe weiterhin eine Diskrepanz gegenüber den deutschen Nationalparken bestehen, die über mehr Personal bei meist erheblich geringerer Fläche verfügen (Liesen/Weber 2018), was in engen Zusammenhang mit der Wahrnehmung der Naturparke als ,Schutzgebiete zweiter Klasse' (vgl. Abbildung 7) in Beziehung zu setzen ist.

Eng verbunden mit der personellen und finanziellen Situation in den Naturparken ist die Möglichkeit, als wichtiger regionaler Akteur in Erscheinung zu treten und ein entsprechendes Ansehen zu entwickeln. Der Abgleich 2011 zu 2020 zeigt, dass Naturparke aus der Sicht ihrer Geschäftsführungen bei den regionalen Entscheidungsgremien an Profil gewinnen konnten, das aber noch ausbaufähig ist. Naturparke brauchen gute Kenntnisse der Region und der Akteursgruppen, um die Belange regionaler Akteure berücksichtigen zu können. Erfolgreiche Naturparkarbeit kann so Schutzbelange mit anderen regionalen Interessen verbinden und im Idealfall dazu beitragen, Parallelstrukturen in einem vielfältigen Akteursspektrum zu vermeiden (vgl. Mehnen/Mose/Strijker 2013; Mehnen/Kabelitz/Liesen 2018). Dies setzt allerdings gleichzeitig die Bereitschaft unterschiedlichster Akteure voraus, die mitunter Projekte selbst aus ihrer Einrichtung heraus umsetzen wollen. Naturparke müssen hier, ihre' Position finden und gegebenenfalls auch behaupten. Je enger vertrauensvolle Kooperationen bzw. gemeinsames Handeln bereits etabliert sind, desto weniger kommt es zu Reibungsverlusten oder Konkurrenzen, wie Beispiele aus den Naturparken Lahn-Dill-Bergland und Dübener Heide im Austausch zwischen den Naturparken und den LEADER-Regionen zeigen (vgl. Klein 2019; Reiss 2019). ${ }^{8}$ Naturparke werden so zu Managern einer nachhaltigen Regionalentwicklung - in einem sich auch thematisch wandelnden und weitenden Feld, wenn etwa an die komplexen Handlungsfelder demographischer Wandel oder Klimawandel gedacht wird (vgl. Gehrlein/von Kutzleben/Düsterhaus et al. 2016). ${ }^{9}$ Die Arbeit der Naturparke ist wiederum noch nicht zwingend in der ,breiten Öffentlichkeit" verankert: Deren Bekanntheitsgrad in der Bevölkerung wird, wie gezeigt, weiterhin als ausbaufähig bewertet, was unter anderem neben der weiteren Positionsbestimmung im

\footnotetext{
8 Vgl. auch weitere Aufsätze in Heft 8/2019 der Zeitschrift LandInForm Spezial: https://www.ble-medienservice.de/7140/ landinform-spezial-8-naturschutz-mit-leader (26.05.2021).

9 Vgl. auch https://www.naturparke.de/aufgaben-ziele/ nachhaltiger-tourismus/katzensprung-kleine-wegegrosse-erlebnisse.html (26.05.2021).
} 
regionalen Akteursnetzwerk - Aufgaben für die kommenden Jahre aufzeigt.

\section{Fazit: Neue Dynamiken durch die Aufgabe der nachhaltigen Regionalentwicklung}

Naturparke haben im Gebietsschutz in Deutschland - wie in unserem Beitrag herausgestellt - sowohl die längste Tradition als auch den höchsten Flächenanteil, sind aber zwischenzeitlich in Bezug auf Bedeutung und Sichtbarkeit in Teilen ins Hintertreffen geraten. Eine neue Gründungswelle nach der deutschen Wiedervereinigung und eine Professionalisierung der Naturparkarbeit, forciert durch den VDN als Dachverband, brachten einen ,frischen Wind' (Weber 2013: $48 \mathrm{ff}$.), der eine starke Flankierung durch die Aufnahme der Aufgabe der nachhaltigen Regionalentwicklung in das Bundesnaturschutzgesetz im Jahr 2002 erhielt. Die Antworten der Naturpark-Geschäftsleitungen zur Aufgabenbewertung in der Befragung durch Weber von 2011 zeugten von einer gewissen Skepsis, was die Frage nach einer aktuellen Positionsbestimmung rund zehn Jahre später im Jahr 2020 bedingte. Welche Schlussfolgerungen - forschungsund praxisorientiert - lassen sich aus den herausgestellten Ergebnissen im Zeitvergleich nun entsprechend ableiten?

Aus einer konzeptionellen Perspektive heraus entsprechen Naturparke mit ihrem vernetzenden Handeln zentralen Kriterien der Regional-governance-Debatte: Prozesse regionaler Selbststeuerung werden gerade bottom-up in netzwerkartiger Regulierung vorangebracht. Da es die Naturpark-Ressourcen alleine nicht zulassen, kommt Kooperationen eine signifikante Bedeutung $\mathrm{zu}$, über die die definierten Ziele erreicht werden sollen. Gleichzeitig bestehen Abhängigkeitsbeziehungen, die eher als ,traditionell ${ }^{\circ}$ top-down-orientiert gedeutet werden können (vgl. Chilla/ Kühne/Neufeld 2016: 259) - hinsichtlich spezifischer Vorgaben durch die Landesregierungen und konkret bezüglich Fördermittel unterschiedlicher Ebenen, die fixen ,Spielregeln' folgen und deren Beantragung durchaus sehr komplex ausfallen kann. Auch die übergreifende Lobbyarbeit durch den VDN schreibt sich ergänzend in ein ,zentralisiertes “ Handeln im Wechselspiel mit den Mitglieds-Naturparken vor Ort ein. Naturparke sind damit - eng eingebunden in verschiedene (übergeordnete) Strukturen - Teil einer multilevel governance (vgl. Pütz/Job 2016: 580). Konzeptionell und praktisch eingeordnet, verbleiben Unschärfen, was unter ,nachhaltige Regionalentwicklung' zu subsumieren ist, eng gekoppelt an die Begriffsweite von ,Nachhaltigkeit". Letztere wird zwar immer wieder definiert und vielfach in politischen Programmen auf verschiedenen Ebenen postuliert (vgl. unter anderem Grunwald/Kopfmüller 2012;
Bundesregierung 2018 sowie die sustainable development goals der Vereinten Nationen), doch die konkrete und stringente Umsetzung bleibt häufig eine große Schwierigkeit. Eine praktische Herangehensweise bietet der VDN seit 2020 seinen Mitgliedern mit einer Arbeitsgruppe ,Regionalentwicklung'. Divergierende Politikinteressen, Herausforderungen bei interdisziplinären Politiken (beispielhaft die Herausforderung einer ,nachhaltigen Agrarpolitik', vgl. z. B. Oppermann/Kasperczyk/Matzdorf et al. 2013; Möckel/Wolf 2020) und damit verbunden fehlgeleitete finanzielle Anreize erschweren nicht nur den Naturparken ihr Handeln. Außerdem sei darauf verwiesen, dass sich Naturparke zahlreichen konkurrierenden Interessenlagen ausgesetzt sehen, die eine Vereinbarkeit ökonomischer, ökologischer und sozialer Belange in Verbindung mit intraund intergenerationeller Gerechtigkeit nicht gerade zum leichten Unterfangen machen. Hinzu kommen Verschiebungen bei den Schwerpunkten, die unter anderem mit der Ausrichtung von Förderprogrammen in Verbindung stehen, das heißt, es wird mitunter auf Themen ,aufgesprungen', für die aktuell Mittel verfügbar sind - also auch dabei eher top-down-induziert. Anwendungsorientiert zeigen die Befragungsergebnisse aus dem Jahr 2020 gleichzeitig, dass die Naturpark-Geschäftsführer/-innen in vielen Bereichen positive Entwicklungsperspektiven konstatieren und sie die Verankerung der Aufgabe der nachhaltigen Regionalentwicklung im Aufgabenportfolio als guten Schritt bewerten.

Künftig wird weiter dafür einzutreten und Lobbyarbeit zu betreiben sein, um die Mindestausstattungen von Naturparken zu verbessern (vgl. auch Forst/Scherfose 2016; Jedicke/ Liesen 2017). Der Veränderungsprozess der Naturparke in Deutschland hin zu Regionalmanagern in ,Modellregionen nachhaltiger Entwicklung' schreitet so weiter voran. Diesen Entwicklungsprozess gilt es zu begleiten und gerade auch Unterschieden zwischen den Naturparken vor dem Hintergrund unterschiedlicher Landesregelungen, Trägerorganisationen etc. noch stärker Rechnung zu tragen. Zudem ist zu beleuchten, inwieweit eher Konkurrenzen oder Kooperationen - gewissen Idealvorstellungen der regional governance folgend - auf regionaler Ebene in Bezug auf Fördermittel oder Sichtbarkeit die Umsetzung einer nachhaltigen Regionalentwicklung im regionalen Kontext bestimmen werden.

Danksagung Wir danken den Geschäftsführerinnen und Geschäftsführern der Naturparke herzlich für die Teilnahme an der durchgeführten Online-Umfrage, zudem den Gutachterinnen, Gutachtern und der Schriftleitung für deren wertvolle Hinweise. Wir bedanken uns darüber hinaus für die Unterstützung durch die Deutsche Forschungsgemeinschaft (DFG) und die Universität des Saarlandes zur Open-Access-Veröffentlichung dieses Beitrags. 


\section{Literatur}

Benz, A.; Dose, N. (Hrsg.) (2010): Governance - Regieren in komplexen Regelsystemen. Eine Einführung. Wiesbaden.

Berr, K.; Jenal, C.; Kühne, O.; Weber, F. (2019): Landschaftsgovernance. Ein Überblick zu Theorie und Praxis. Wiesbaden. https://doi.org/10.1007/978-3-658-27484-9

Böcher, M. (2006): Regional Governance: Ein Konzept im Spannungsverhältnis zwischen Anspruch und Wirklichkeit. In: von Blumenthal, J.; Bröchler, S. (Hrsg.): Von Government zu Governance. Analysen zum Regieren im modernen Staat. Hamburg, 119-141.

Böcher, M. (2018): Regional Governance und Großschutzgebiete. In: Weber, F.; Weber, F.; Jenal, C. (Hrsg.): Wohin des Weges? Regionalentwicklung in Großschutzgebieten. Hannover, 56-71. = Arbeitsberichte der ARL 21.

Borsdorf, A.; Jungmeier, M.; Braun, V.; Heinrich, K. (Hrsg.) (2020): Biosphäre 4.0. UNESCO Biosphere Reserves als Modellregionen einer nachhaltigen Entwicklung. Berlin. https://doi.org/10.1007/978-3-66260707-7

Brackhane, S.; Liesen, J.; Bieber, M.; Godt, J.; Schoof, N.; Rosenthal, G.; Reif, A. (2019): Flächenpotenziale und Entwicklungskonzepte zur Realisierung des $2 \%$-Wildnisziels in Naturparken in Deutschland. In: Natur und Landschaft 94, 9/10, 402-408. https://doi.org/10.17433/ 9.2019.50153733.402-408

Bröchler, S.; von Blumenthal, J. (2006): Von Government zu Governance - Analysen zu einem schwierigen Verhältnis. In: von Blumenthal, J.; Bröchler, S. (Hrsg.): Von Government zu Governance. Analysen zum Regieren im modernen Staat. Hamburg, 7-21.

Bundesregierung (2018): Deutsche Nachhaltigkeitsstrategie. Aktualisierung 2018. Berlin.

Chilla, T.; Kühne, O.; Neufeld, M. (2016): Regionalentwicklung. Stuttgart.

Erdmann, K.-H. (1998): Nachhaltige Entwicklung als regionale Perspektive. In: Heinritz, G.; Wießner, R.; Winiger, M. (Hrsg.): Nachhaltigkeit als Leitbild der Umwelt- und Raumentwicklung in Europa. Stuttgart, 90-95.

EUROPARC Deutschland (2013): Bündnisse für die Natur. Wege zu einem funktionalen Schutzgebietssystem in Deutschland. Berlin.

Forst, R. (2019): Entwicklung, Stand und Herausforderungen der Naturparkarbeit in Deutschland. In: Natur und Landschaft 94, 9/10, 374-381. https://doi.org/10.17433/ 9.2019.50153725.374-381

Forst, R.; Scherfose, V. (2016): Vorschläge für MindestStandards deutscher Naturparke. In: Forst, R.; Scherfose, V. (Hrsg.): Naturparkmanagement in Deutschland
- Qualitätsoffensive Naturparke. Bonn-Bad Godesberg, 233-245. = Naturschutz und Biologische Vielfalt 55.

Fürst, D. (2001): Regional governance - ein neues Paradigma der Regionalwissenschaften? In: Raumforschung und Raumordnung 59, 5-6, 370-380. https://doi.org/10. 1007/BF03183038

Fürst, D. (2010): Regional Governance. In: Benz, A.; Dose, N. (Hrsg.): Governance - Regieren in komplexen Regelsystemen. Eine Einführung. Wiesbaden, 49-68.

Gehrlein, U.; von Kutzleben, N.; Düsterhaus, B.; Niclas, G. (Hrsg.) (2016): Der demografische Wandel und seine Wirkung auf Großschutzgebiete. Bonn-Bad Godesberg. = BfN-Skripten 429 .

Gehrlein, U.; Mathias, C.; Steil, C. (2019): Strukturelle Voraussetzungen einer erfolgreichen Naturparkarbeit. Schlussfolgerungen aus der Evaluation der baden-württembergischen Naturparke. In: Natur und Landschaft 94, 9/10, 390-395. https://doi.org/10.17433/9.2019. 50153729.390-395

Grunwald, A.; Kopfmüller, J. (2012): Nachhaltigkeit. Eine Einführung. Frankfurt am Main.

Horstick, A.; Schubert, S. (2019): Naturparke als Akteure zur Umsetzung der Bildung für nachhaltige Entwicklung. In: Natur und Landschaft 94, 9/10, 409-415. https://doi. org/10.17433/9.2019.50153735.409-415

Isbary, G. (1959): Gutachten über geeignete Landschaften für die Auswahl von Naturparken vom Standpunkt der Raumordnung. Bonn-Bad Godesberg.

Jedicke, E.; Liesen, J. (2017): Naturparke und Naturschutz Leistungen, Potenziale und Perspektiven. In: BBN - Bundesverband Beruflicher Naturschutz (Hrsg.): Naturschutz und Landnutzung - Analysen, Diskussionen, zeitgemäße Lösungen. Bonn, 28-37. = Jahrbuch für Naturschutz und Landschaftspflege 61.

Job, H. (1991): Freizeit und Erholung mit oder ohne Naturschutz? Bad Dürkheim. = Pollichia 22.

Job, H.; Metzler, D. (2006): Naturparks + Tourismus = Regionalentwicklung? In: Natur und Landschaft 81, 7, 355-361.

Job, H.; Woltering, M.; Warner, B.; Heiland, S.; Jedicke, E.; Meyer, P.; Nienaber, B.; Plieninger, T.; Pütz, M.; Rannow, S.; von Ruschkowski, E. (2016): Biodiversität und nachhaltige Landnutzung in Großschutzgebieten. In: Raumforschung und Raumordnung 74, 6, 481-494. https://doi.org/10.1007/s13147-016-0440-5

Kaether, J. (1994): Großschutzgebiete als Instrumente der Regionalentwicklung. Hannover. $=$ Arbeitsmaterial der ARL 210.

Klein, M. (2019): Raum für gemeinsame Visionen. LahnDill-Bergland. In: LandInForm Spezial 8, 16-17.

Köster, U. (2019): Weiterentwicklung und Fortschreibung der Aufgaben und Ziele für die Naturparke in Deutsch- 
land. In: Natur und Landschaft 94, 9/10, 436-441. https://doi.org/10.17433/9.2019.50153743.436-441

Kühne, O. (2011): Heimat und sozial nachhaltige Landschaftsentwicklung. In: Raumforschung und Raumordnung 69, 5, 291-301. https://doi.org/10.1007/s13147011-0108-0

Kühne, O.; Meyer, W. (2015): Gerechte Grenzen? Zur territorialen Steuerung von Nachhaltigkeit. In: Kühne, O.; Weber, F. (Hrsg.): Bausteine der Regionalentwicklung. Wiesbaden, 25-40. https://doi.org/10.1007/978-3-65802881-7_3

Liesen, J.; Coch, T. (2015): Finanzielle Unterstützung des Landschaftserhalts durch die Kurtaxe. Erfahrungen aus der Ferienregion Münstertal-Staufen (Naturpark Südschwarzwald). In: Naturschutz und Landschaftsplanung 47, 3, 69-76.

Liesen, J.; Weber, F. (2018): Regionalentwicklung im Spannungsfeld von Naturpark Saar-Hunsrück und Nationalpark Hunsrück-Hochwald. In: Weber, F.; Weber, F.; Jenal, C. (Hrsg.): Wohin des Weges? Regionalentwicklung in Großschutzgebieten. Hannover, 122-150. = Arbeitsberichte der ARL 21.

Liesen, J.; Schäfer, A. (2019): Qualität zählt! - Vorteile und Nutzen der Qualitätsoffensive Naturparke. In: Natur und Landschaft 94, 9/10, 396-401. https://doi.org/10.17433/ 9.2019.50153731.396-401

Liesen, J.; Hoheisel, D.; Mengel, A.; Köster, U. (2017): Entwicklungsperspektiven für die Naturparkplanung. Resultate der Qualitätsoffensive Naturparke und einer Befragung. In: Naturschutz und Landschaftsplanung 49, 11 , 355-362.

Liesen, J.; Köster, U.; Porzelt, M. (2008): 50 Jahre Naturparke in Deutschland. Das Petersberger Programm der Naturparke setzt internationale Ziele zum Erhalt der biologischen Vielfalt. In: Naturschutz und Landschaftsplanung 40, 1, 26-32.

Lommel, E. (1974): Naturparke in Deutschland. In: Stöhr, R. (Hrsg.): Ideen und Taten: Alfred Toepfer zum 80. Geburtstag. Hamburg, 95-112.

Majewski, L.; Engelbauer, M.; Job, H. (2019): Tourismus und nachhaltige Entwicklung in deutschen Naturparken. In: Natur und Landschaft 94, 9/10, 422-426. https://doi. org/10.17433/9.2019.50153739.422-426

Mayer, M.; Stoll-Kleemann, S. (2020): Tourismus und Regionalentwicklung innerhalb und außerhalb ostdeutscher Großschutzgebiete. In: Becker, S.; Naumann, M. (Hrsg.): Regionalentwicklung in Ostdeutschland. Dynamiken, Perspektiven und der Beitrag der Humangeographie. Berlin, 481-495. https://doi.org/10.1007/978-3662-60901-9_37

Mehnen, N.; Kabelitz, S.; Liesen, J. (2018): Akteure und Akteurskonstellationen in Naturparken: Wer agiert und kooperiert? In: Weber, F.; Weber, F.; Jenal, C. (Hrsg.): Wohin des Weges? Regionalentwicklung in Großschutzgebieten. Hannover, 96-121. = Arbeitsberichte der ARL 21.

Mehnen, N.; Mose, I.; Strijker, D. (2013): Governance and Sense of Place: Half a Century of a German Nature Park. In: Environmental Policy and Governance 23, 1, 46-62. https://doi.org/10.1002/eet.1592

Möckel, S.; Wolf, A. (2020): Düngung bleibt weiterhin eine ökologische, rechtliche und politische Herausforderung. In: Natur und Recht 42, 11, 736-746. https://doi.org/10. 1007/s10357-020-3758-2

Mose, I. (2009): Akzeptanz, Einstellung und Image als Einflussgrößen von Großschutzgebieten: Einige theoretische und methodische Vorüberlegungen. In: Mose, I. (Hrsg.): Wahrnehmung und Akzeptanz von Großschutzgebieten. Oldenburg, 9-35. = Wahrnehmungsgeographische Studien 25 .

Mose, I.; Hammer, T.; Siegrist, D.; Weixlbaumer, N. (2019): Gebietsschutz in Europa - Herausforderungen für wissenschaftliche Kooperation. Erfahrungen der Forschergruppe NeReGro. In: Raumforschung und Raumordnung | Spatial Research and Planning 77, 1, 81-93. https://doi. org/10.2478/rara-2019-0004

Naturpark Südschwarzwald (2018): Naturpark-Plan 2025. Feldberg.

Nienaber, B. (2018): Partizipation in Großschutzgebieten - untersucht am Beispiel der Beteiligung an den Workshops zur Erstellung des Rahmenkonzeptes des Biosphärenreservates Bliesgau 2014. In: Weber, F.; Weber, F.; Jenal, C. (Hrsg.): Wohin des Weges? Regionalentwicklung in Großschutzgebieten. Hannover, 192-205. = Arbeitsberichte der ARL 21.

Oppermann, R.; Kasperczyk, N.; Matzdorf, B.; Reutter, M.; Meyer, C.; Kuick, R.; Stein, S.; Ameskamp, K.; Gelhausen, J.; Bleil, R. (2013): Reform der Gemeinsamen Agrarpolitik (GAP) 2013 und Erreichung der Biodiversitätsund Umweltziele. Bonn-Bad Godesberg. = Naturschutz und Biologische Vielfalt 135.

Pütz, M.; Job, H. (2016): Governance und Regionalentwicklung in Großschutzgebieten der Schweiz und Österreichs. In: Raumforschung und Raumordnung 74, 6, 569-583. https://doi.org/10.1007/s13147-016-0451-2

Reiss, U. (2019): Teamwork über Ländergrenzen hinweg. Dübener Heide. In: LandInForm Spezial 8, 14-15.

Rhodes, R. (1996): The New Governance: Governing without Government. In: Political Studies 44, 4, 652-667. https://doi.org/10.1111/j.1467-9248.1996.tb01747.x

Risthaus, K.; Schubert, S. (2017): Faszination Natur erleben in den Nationalen Naturlandschaften. In: BBN - Bundesverband Beruflicher Naturschutz (Hrsg.): Naturschutz und Landnutzung - Analysen, Diskussionen, zeitgemäße 
Lösungen. Bonn, 180-187. = Jahrbuch für Naturschutz und Landschaftspflege 61.

Scherfose, V. (2019): Schutzgebietsanteile der deutschen Naturparke und Überlegungen zur Weiterentwicklung der Naturpark-Gebietskulissen. In: Natur und Landschaft 94, 9/10, 427-435. https://doi.org/10.17433/9. 2019.50153741.427-435

Schöbel, S. (2018): Einleitung. In: Schöbel, S. (Hrsg.): Landschaftsvertrag. Zur kritischen Rekonstruktion der Kulturlandschaft. Berlin, 9-23.

Schöttle, R.; Cammerer, C. (2019): Rolle, Leistungen und Perspektiven im Bereich der nachhaltigen Regionalentwicklung am Beispiel des Naturparks Südschwarzwald. In: Natur und Landschaft 94, 9/10, 416-421. https://doi. org/10.17433/9.2019.50153737.416-421

Schubert, H. (2004): Netzwerkmanagement - Planung und Steuerung von Vernetzung zur Erzeugung raumgebundenen Sozialkapitals. In: Müller, B.; Löb, S.; Zimmermann, K. (Hrsg.): Steuerung und Planung im Wandel. Festschrift für Dietrich Fürst. Wiesbaden, 177-200.

VDN - Verband Deutscher Naturparke (2009): Naturparke in Deutschland: Aufgaben und Ziele. Bonn.

VDN - Verband Deutscher Naturparke (2017a): Landschaften voller Leben. Ergebnisse des Projektes „Europe's Nature, Regional and Landscape Parks“. Bonn.

VDN - Verband Deutscher Naturparke (2017b): Landschaften voller Leben. Europas Natur-, Regional- und Landschaftsparke - Modellregionen für die nachhaltige Entwicklung ländlicher Räume. Bonn.

VDN - Verband Deutscher Naturparke (2018a): Naturparke 2030 - Wartburger Programm der Naturparke in Deutschland. Bonn. https://www.naturparke.de/fileadmin/files/ public/Aufgaben_und_Ziele/PDF/Wartburger_Programm mit_Logo.pdf (25.05.2021).

VDN - Verband Deutscher Naturparke (2018b): Wildnisgebiete und große Prozessschutzflächen in Naturparken. Ein Handlungsleitfaden. Bonn.
VDN - Verband Deutscher Naturparke (2019): Naturparkplanung. Ein Leitfaden für die Praxis. Bonn.

VDN - Verband Deutscher Naturparke (2020): Naturparke in Deutschland 2030. Aufgaben und Ziele. Bonn.

von Ruschkowski, E.; Nienaber, B. (2016): Akzeptanz als Rahmenbedingung für das erfolgreiche Management von Landnutzungen und biologischer Vielfalt in Großschutzgebieten. In: Raumforschung und Raumordnung 74, 6, 525-540. https://doi.org/10.1007/s13147-016-0429-0

Weber, F. (2013): Naturparke als Manager einer nachhaltigen Regionalentwicklung. Probleme, Potenziale und Lösungsansätze. Wiesbaden. https://doi.org/10.1007/ 978-3-658-01972-3

Weber, F.; Weber, F. (2014): Naturparke als Regionalmanager - Instrumente einer grenzüberwindenden und „nachhaltigen“ Regionalentwicklung?! In: Grotheer, S.; Schwöbel, A.; Stepper, M. (Hrsg.): Nimm's sportlich Planung als Hindernislauf. Hannover, 48-61. = Arbeitsberichte der ARL 10.

Weber, F.; Weber, F. (2015): Naturparke und die Aufgabe der nachhaltigen Regionalentwicklung. Jenseits von Wanderwegemarkierern und Parkbankaufstellern. In: Naturschutz und Landschaftsplanung 47, 5, 149-156.

Weber, F.; Weber, F. (2019): Naturparke - Biosphärenreservate - Nationalparke: Schlaglichter auf ,Großschutzgebietskonfliktlandschaften' zu Beginn des 21. Jahrhunderts. In: Berr, K.; Jenal, C. (Hrsg.): Landschaftskonflikte. Wiesbaden, 247-268. https://doi.org/10.1007/978-3658-22325-0_16

Weixlbaumer, N.; Siegrist, D.; Mose, I.; Hammer, T. (2015): Großschutzgebiete als Instrumente für zukunftsorientierte Regionalentwicklung in Europa - die Sicht der Schutzgebietsverantwortlichen und Forscher am Beispiel von Partizipation und Regional Governance. In: Erdmann, K.-H.; Bork, H.-R.; Job, H. (Hrsg.): Naturschutz in geographischer Perspektive. Bonn-Bad Godesberg, 81-88. = BfN-Skripten 400. 